Dom. Cien., ISSN: 2477-8818

Vol. 4, núm. 1, enero, 2018, pp. 235-248

Objetos de aprendizaje para la enseñanza de las ecuaciones en matemáticas preuniversitarias, SEGUNDA PARTE

\title{
Objetos de aprendizaje para la enseñanza de las ecuaciones en matemáticas preuniversitarias, SEGUNDA PARTE
}

\section{Learning objects for the teaching of equations in pre-university mathematics, SECOND PART}

\author{
Aprendendo objetos para o ensino de equações em matemática pré- \\ universitária, SEGUNDA PARTE
}

\author{
Mario R. Salvador-Giler ${ }^{\mathrm{I}}$ \\ mario.salvado@gmail.com \\ Tania M. Ferrín- Menéndez II \\ tania.ferrin1@gmail.com \\ Homero M. Ferrín-Schett ${ }^{\mathrm{III}}$ \\ homero.ferri32@gmail.com
}

Recibido: 10 de septiembre de 2017 * Corregido: 25 de noviembre de 2017 * Aceptado: 27 de diciembre de 2017

I. Diploma Superior en Economía Internacional, Diploma Superior en Economía del Ecuador y del Mundo, Magister en Negocios Internacionales y Gestión de Comercio Exterior, Ingeniero Comercial, Universidad de Guayaquil, Ecuador.

II. Licenciada en Contabilidad y Auditoría, Contadora Pública, Economista, Universidad de Guayaquil, Ecuador.

III. Diploma Superior en Economía Internacional, Diploma Superior en Economía del Ecuador y del Mundo, Diploma Superior en Gestión Empresarial Internacional, Magister en Negocios Internacionales y Gestión de Comercio Exterior, Ingeniero Civil, Arquitecto, Universidad de Guayaquil, Ecuador. 


\section{Resumen}

El objeto de aprendizaje (OA) ecuación, constituye una alternativa para la enseñanza de este concepto en la Unidad Curricular Matemática Preuniversitaria de las Universidades Ecuatorianas, en razón de la necesidad de nuevas estrategias de enseñanza, dado los cambios presentados en la estructura curricular de las instituciones educativas antes mencionadas y el creciente desarrollo de las tecnologías de la información y comunicación. La innovación en tecnología y la sociedad demandante, han ocasionado que las universidades incurran en la prestación servicios educativos, como parte de programas de desarrollo sustentable. Así pues, la educación virtual viene a representar el medio por el cual se propician diálogos didácticos entre los actores involucrados en la formación virtual. En este sentido el objeto de aprendizaje como fenómeno de investigación emergente, se posiciona en el proceso de enseñanza como un recurso con características específicas, para ser introducido en la educación del presente siglo. El material didáctico que se presenta en esta segunda parte del presente artículo, se constituye en una alternativa ante el método tradicional de enseñanza; que le permitirá al estudiante de matemática preuniversitaria construir su propio aprendizaje, a través de la aplicación de estrategias que facilitan su participación activa, y al docente afianzar el verdadero rol de facilitador. Palabras clave: objeto de aprendizaje; ecuación; educación virtual; material didáctico.

\section{Abstract}

The object of learning (OA) equation is an alternative for the teaching of this concept in the Preuniversity Mathematics Curriculum Unit of the Ecuadorian Universities, due to the need for new teaching strategies, given the changes presented in the curricular structure of the educational institutions mentioned above and the growing development of information and communication technologies. Innovation in technology and the demanding society have caused universities to provide educational services as part of sustainable development programs. Thus, virtual education comes to represent the means by which didactic dialogues are facilitated among the actors involved in virtual training. In this sense, the object of learning as an emerging research phenomenon is positioned in the teaching process as a resource with specific characteristics, to be introduced in the education of the present century. The didactic material presented in this second part of this article is an alternative to the traditional teaching method; that will allow the pre-university math student to build their own 
learning, through the application of strategies that facilitate their active participation, and the teacher to strengthen the true role of facilitator.

Keywords: object of learning; equation; virtual education; teaching materials.

\section{Resumo}

O equação do objeto de aprendizagem (OA) é uma alternativa para o ensino deste conceito na Unidade Curricular da Universidade Pré-Universitária das Universidades Equatorianas, devido à necessidade de novas estratégias de ensino, atendendo às mudanças apresentadas na estrutura curricular da instituições educacionais mencionadas acima e o crescente desenvolvimento das tecnologias da informação e da comunicação. A inovação em tecnologia e a sociedade exigente fizeram com que as universidades ofereçam serviços educacionais como parte de programas de desenvolvimento sustentável. Assim, a educação virtual vem representando os meios pelos quais os diálogos didáticos são facilitados entre os atores envolvidos no treinamento virtual. Nesse sentido, o objetivo da aprendizagem como fenômeno de pesquisa emergente é posicionado no processo de ensino como um recurso com características específicas, a serem introduzidas na educação do presente século. $\mathrm{O}$ material didático apresentado nesta segunda parte deste artigo é uma alternativa ao método de ensino tradicional; que permitirá que o estudante de matemática pré-universitário construa sua própria aprendizagem, através da aplicação de estratégias que facilitam sua participação ativa e do professor para fortalecer o verdadeiro papel do facilitador.

Palavras chave: objeto de aprendizagem; equação; educação virtual; material didático.

\section{Introducción}

Asumir el compromiso de desarrollar una actividad, que permita a los estudiantes adquirir nuevos conocimientos, requiere de la verdadera vocación del docente. Convertida ésta en la acción bajo ciertas premisas teóricas, determinadas por las experiencias y creencias del docente en su cotidianidad. Más allá de ello, el profesor de matemáticas debe percibir al estudiante como la razón de ser de su acción. En este sentido Schon (1998) en Miguez (2010), indica que la ausencia de esta percepción, hace que el docente pierda la noción de lo que debe ofrecer como profesor de matemáticas, socava la noción de su actividad profesional. 
En la actualidad, el acto educativo ha sido alcanzado por la denominada educación virtual, como modalidad de estudio alternativa debido a los avances psicopedagógicos y la creciente y constante innovación tecnológica, y han dado paso a la oleada de propuestas educativas en línea, debido a la globalización y al uso de las tecnologías de la información y la comunicación. De allí que la enseñanza virtual y la construcción de objetos de aprendizaje (OA), representan fenómenos de estudio permanente, especialmente en las áreas de formación integral.

La elaboración de este objeto de aprendizaje toma en cuenta la convergencia entre métodos, tecnologías, aplicaciones y servicios orientados a sustentar y facilitar la enseñanza y aprendizaje vía internet. Para la construcción del mismo se toma en cuenta los aspectos relacionados a: teorías de la enseñanza constructivista del conocimiento, desarrollo de habilidades en el manejo de plataformas tecnológicas, desarrollo de materiales didácticos y desarrollo de objetos de aprendizaje.

Lo anteriormente expuesto, permite presentar en este artículo el material didáctico, para ser utilizado en plataformas tecnológicas dedicadas a la enseñanza y el aprendizaje, a ser dirigido a estudiantes de matemática preuniversitaria, susceptible a ser reformulado ya que una de las características de los objetos de aprendizaje, es la de ser reutilizados con el fin de lograr alcanzar en los participantes de la educación matemática, el mayor de los conocimientos en cuanto a conceptos básicos aplicables en su formación profesional.

\section{Estructura didáctica del objeto de aprendizaje}

En este apartado se presentará el material dada la estructura didáctica del objeto de aprendizaje, que puede ser reemplazada en diferentes formatos, según el ambiente de la plataforma tecnológica utilizada. Según Guardoño (2011), presentar un objeto de aprendizaje, pretende en corto plazo una baja inversión derivada de la reutilización de tales objetos. 
Nombre del Objeto de Aprendizaje: Ecuación.

Objeto de Aprendizaje correspondiente a la Solicitud número:

Estructura Didáctica (En cada espacio se debe escribir el nombre del fichero físico a entregar que contiene la información referente al aspecto. Si la información es breve puede incluirse en el espacio correspondiente de la planilla):

\section{Intencionalidad formativa:}

Este objeto de aprendizaje, está referido a la definición de ecuación, siendo la ecuación un concepto básico central aplicable en el cálculo infinitesimal, el cual, hace referencia al tema de funciones reales y el límite de una función.

\section{Contenido:}

* Conceptos Básicos:

Igualdad.

- Identidad.

- Incógnitas.

* Definición de Ecuación.

* Soluciones o Raíces de una Ecuación.

* Ecuación Equivalente

* Transposición de Términos.

* Grado de una Ecuación.

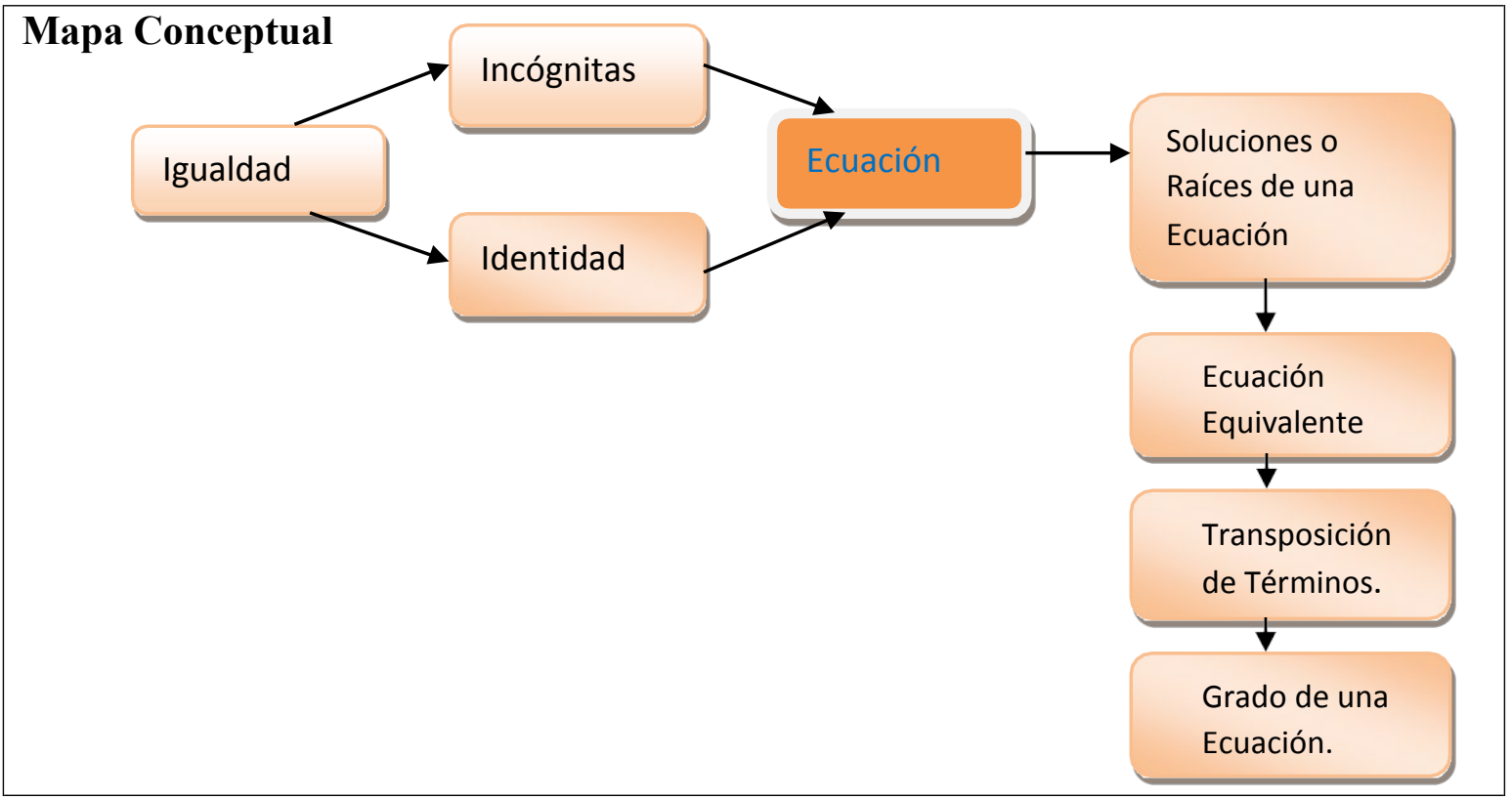




\section{Desarrollo del contenido}

\section{Introducción:}

El propósito de desarrollar este objeto de aprendizaje, es que sirva de apoyo a la instrucción para la Subunidad Curricular Ecuación, aquí se proporciona de manera clara las definiciones básicas que están contenidas en la definición de ecuación, en forma teórica y práctica, que luego será aplicada en la resolución de ejercicios y problemas relacionados con el contenido tratado.

\section{Objetivos:}

General:

- Estudiar la definición de Ecuación.

\section{Específicos:}

- Reconocer el significado del lenguaje matemático.

- Distinguir las expresiones numéricas y algebraicas.

- Resolver ecuaciones.

\section{Definición:}

La palabra ecuación, deriva del latín aequatio, que significa igualdad. Para las matemáticas, una ecuación es una igualdad que contiene una o más incógnitas.

Ahora bien, como el objetivo principal de este espacio es definir la palabra en referencia, se hace necesario hacer referencia a otros dos conceptos básicos, como son el de igualdad y el de identidad.

Una igualdad es una expresión que se obtiene al igualar dos cantidades que tienen el mismo valor, para ello se utiliza el signo $=$, que se lee, igual.

Por ejemplo,

1. $(x+y)^{2}=x^{2}+2 x y+y^{2}$

2. $3 x+2=x+5$

Ambas son igualdades.

Observación: En una igualdad todo lo que esté a la izquierda del signo igual, se llama primer miembro, y todo que esté a la derecha, segundo miembro. La igualdad 1. Es cierta para cualesquiera valores de $\mathbf{x}$ y $\mathbf{y}$, pero la igualdad 2. No se satisface para todo valor de

x. De acuerdo a este hecho podemos clasificar las igualdades en identidades y ecuaciones. Una identidad es una igualdad en la que ambos miembros son iguales para todos los valores de las variables para los cuales están definidos los miembros. Es el caso de la igualdad 1.

Una ecuación es una igualdad en la cual ambos miembros son iguales sólo para valores particulares de las variables. Es el caso de la igualdad 2. 


$$
\begin{aligned}
& \text { Ejemplos para identificar identidades } y \text { ecuaciones. } \\
& \text { 1.- } 2(x+4)=8 \\
& \text { 2.- }(x+2)^{2}=x^{2}+4 \\
& \text { 3.- } 3(2 x-2 / 3)=6 x-2 \\
& \text { 4.- } x^{2}-9=(x+3)(x-3) \\
& \text { 5.- }(x+2 y)(x-2 y)-(x-2 y)^{2}+4 y(2 y-x)=0
\end{aligned}
$$

\section{Solución:}

Las igualdades (3), (5), (4) son identidades. Se satisfacen para cualquier valor de x. En cada uno de estos casos, el segundo miembro se obtiene a partir de una sencilla transformación del primero.

Las igualdades (2) y (1) son ecuaciones. Ambas son ciertas sólo para x=0.

\section{¿Qué es una ecuación?}

Una ecuación es una igualdad entre expresiones algebraicas en la cual ambos miembros son iguales sólo para valores particulares de las variables.

Notación:

$a x+b=0$, donde a y $b$ son diferentes de cero.

\section{Responda a estas preguntas:}

a) $3 x+7=10$

Esta igualdad sólo es cierta si: $\mathrm{x}=$

b) $(7 / 2) \mathrm{x}+4-12$

Esta igualdad sólo es cierta six $=$

c) $-7 \mathrm{x}+4=10$

Esta igualdad sólo es cierta si: $x=$

d) $9 x+3=13$

Esta igualdad sólo es cierta si: $\mathrm{x}=$

e) $(8 / 3) \mathrm{x}-421$

Esta igualdad sólo es cierta si: $\mathrm{x}=$

\section{Elementos de una ecuación:}

MIEMBROS: son las expresiones que aparecen a cada lado del signo $=$.

Se conoce como miembros a las expresiones algebraicas que presentan los datos (valores conocidos) y las incógnitas (valores desconocidos), relacionados a través de operaciones matemáticas.

\section{TÉRMINOS: son los monomios de cada miembro.}


Los datos presentados en una ecuación pueden ser números, constates, coeficientes o variables.

INCÓGNITAS: Son las letras que aparecen en la ecuación.

Las incógnitas son las variables cuyos valores son desconocidos en una ecuación, y suelen identificarse con las últimas letras del alfabeto, que reemplazan al valor que se intenta hallar.

GRADO DE LA ECUACIÓN: es el mayor exponente con que figura la incógnita (una vez realizadas todas las operaciones).

SOLUCIONES: son los valores que deben tener las incógnitas para que la igualdad entre los miembros sea cierta.

Aquí se coloca una ecuación en donde se señalen cada uno de sus elementos $4 x-$ $3=\mathbf{2}+\mathbf{1}$

\section{¿Cómo se resuelve una ecuación?}

Resolver una ecuación significa determinar el o los valores de las incógnitas que las satisfacen.

Se puede decir, que las soluciones o raíces de una ecuación son los valores de las incógnitas que la verifican.

En la ecuación $\mathbf{x}-2=4$, la letra $x$ es la incógnita, y la solución o raíz de la ecuación es $\mathbf{x}=6$.

Pues,

$\mathbf{x}=\mathbf{4}+\mathbf{2}$

\section{Método para resolver una ecuación:}

El método general para resolver una ecuación, consiste en transformarla en otra ecuación equivalente, cuyas raíces y soluciones puedan obtenerse con más facilidad que las de la ecuación dada.

De allí que, dos ecuaciones son equivalentes cuando tienen las mismas soluciones.

\section{Ejemplos:}

1.- Las ecuaciones:
a) $x-2=0 y$
b) $2 x=4$ son equivalentes, ya que ambas tienen como única raíz al número $x=2$.

\section{Solución:}
a) $\mathrm{x}=\mathbf{2}$
b) $x=4 / 2$

2.- Las ecuaciones:

a) $x-2=0 y$ 
b) $\mathbf{x}^{2}-4=0$ no son equivalentes, porque la primera ecuación tiene como única raíz al número 2, mientras que la segunda tiene dos raíces, \pm 2 .

Solución:
a) $\mathbf{x}=\mathbf{2}$
b) $x^{2}=4$
$x= \pm 2$

\section{A continuación consideraremos las operaciones que pueden efectuarse en una ecuación dada para obtener una ecuación equivalente.}

1) Si se suma o se resta una misma expresión a ambos miembros de una ecuación, la expresión resultante es equivalente a la dada.

2) Si ambos miembros de una ecuación, se multiplican por, o se dividen entre la misma constante no nula, la ecuación resultante es equivalente a la dada.

\section{Ejemplo:}

Consideremos la ecuación $4 x-7=14$.

Procedamos a resolverla:

Ecuación dada: $\mathbf{4 x - 7 = 1 4}$

Sumando 7 a ambos miembros: $\mathbf{4 x - 7 + 7 = 1 4 + 7}$

Dividiendo ambos miembros por $4: 4 x / 4=21 / 4$

Entonces la solución de $4 x-7=14$, es: $x=21 / 4$

A grosso modo, al resolver una ecuación, la estrategia general es modificarla paso a paso hasta llegar a una forma en que la solución sea obvia. Desde luego, hay que tener cuidado al hacer las modificaciones para no cambiar las soluciones.

Importante:

Cuando no se está en presencia de ecuaciones equivalentes, se pueden presentar los casos en que las ecuaciones reciben el nombre de ecuación redundante y de ecuación defectuosa.

Ecuación redundante: $\mathrm{Si}$ ambos miembros de una ecuación dada se multiplican por una expresión que contenga la variable, la nueva ecuación puede tener una o más raíces que no son soluciones de la ecuación dada. Estas nuevas raíces se denominan raíces extrañas y la nueva ecuación se llama redundante con respecto a la ecuación dada.

\section{Ejemplo:}

Dada la ecuación:

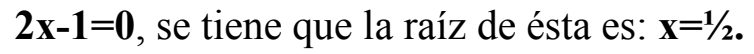

Si multiplicamos a ambos miembros de la ecuación por (x-8), obtenemos la ecuación:

$(2 \mathrm{x}-1)(\mathrm{x}-8)=\mathbf{0}$.

Esta ecuación presenta las raíces: $\mathbf{x}=1 / 2 \mathbf{y} \mathbf{x}=\mathbf{8}$. 
Por tanto:

Las ecuaciones: $\mathbf{2 x - 1}=\mathbf{0}$ y $(\mathbf{2 x - 1})(\mathbf{x}-\mathbf{8})=\mathbf{0}$.

no son equivalentes.

Pues, la raíz $\mathbf{x}=\mathbf{8}$ es extraña a la ecuación: $\mathbf{2 x - 1}=\mathbf{0}$,

Se puede concluir que la ecuación: $(2 \mathbf{x}-1)(\mathbf{x}-8)=0$ es redundante con respecto a la ecuación: $\mathbf{2 x - 1 = 0 .}$

Ecuación defectuosa: Si ambos miembros de una ecuación dada se dividen entre una misma expresión que contenga la variable, la nueva ecuación puede tener una raíz, mas o menos raíces que la ecuación original. En este caso se dice que la nueva ecuación es defectuosa con respecto a la ecuación dada.

\section{Ejemplo:}

1.- Se tiene la ecuación: $(\mathbf{2 x - 1})(\mathbf{x}-\mathbf{8})=\mathbf{0}$

dividamos ambos miembros de la ecuación entre $\mathbf{x - 8}$.

Al hacerlo, se obtiene la ecuación: $\mathbf{2 x - 1}=\mathbf{0}$.

Luego, la ecuación: $(\mathbf{2 x - 1})(\mathbf{x}-\mathbf{8})=\mathbf{0}$, tiene raíces: $\mathbf{x}=1 / 2 \mathbf{2} \mathbf{y}=\mathbf{8}$,

Se puede concluir que la ecuación: $\mathbf{2 x - 1}=\mathbf{0}$, es defectuosa con respecto a la ecuación $(\mathbf{2} \mathbf{x}-$ $\mathbf{1})(\mathbf{x}-\mathbf{8})=\mathbf{0}$, pues la ecuación $\mathbf{2 x - 1}=\mathbf{0}$ sólo tiene raíz $\mathbf{x}=1 / 2$.

2.- Sea la ecuación: $\mathbf{x = 5}$.

Elevemos al cuadrado ambos miembros de la ecuación, obtenemos $\mathbf{x}^{\mathbf{2}}=\mathbf{2 5}$ cuyas raíces son: $\mathbf{x}= \pm \mathbf{5}$.

Lo que significa que esta operación ha introducido la raíz extraña: $\mathbf{x = - 5}$.

Nota:

No todas las ecuaciones se resuelven utilizando exclusivamente las operaciones 1) y 2) que hemos indicado. Ello depende del tipo de expresiones (polinómicas, racionales, logarítmicas, exponenciales, trigonométricas, etc.) asociadas a la ecuación. Pero en todo caso las operaciones antes mencionadas siempre serán válidas. Para evitar resultados incorrectos, conviene que el estudiante tome como norma la comprobación de cada raíz en la ecuación original, por sustitución directa.

\footnotetext{
Ejemplos para identificar ecuaciones equivalentes, defectuosas o redundantes. Dada la ecuación: $\mathbf{x}^{\mathbf{3}} \mathbf{- 4 x}=\mathbf{0}$, reconocer cuales de ellas cumple alguna de las tres características descritas anteriormente.

1.- $x^{3}-2 x+3=2 x+3$

2.- $x^{2}-4=0$

3.- $x^{3}=4 x$

4.- $x^{3}(x+3)=4 x(x+3)$

5.- $x(x-2)(x+2)=0$

6.- $x(x-2)=0$

7.- $\left(x^{3}-4 x\right)(x-5)=0$
} 


\section{Solución:}

Son equivalentes las opciones (1), (3) y (5).

Cada una de éstas ecuaciones puede deducirse a partir de $\mathbf{x}^{\mathbf{3}} \mathbf{- 4 x}=\mathbf{0}$, efectuando

transformaciones adecuadas para obtener una ecuación equivalente.

Son defectuosas las opciones (2) y (6).

La ecuación (2) se obtiene al dividir ambos miembros de $\mathbf{x}^{\mathbf{3}} \mathbf{- 4 x}=\mathbf{0}$ por $x$; y la (6), al dividir por $\mathbf{x}-2$. Fíjese que ambos casos, se pierden soluciones.

Son redundantes (4) y (7).

Cada una de ellas se obtiene al multiplicar los miembros de $\mathbf{x}^{\mathbf{3}} \mathbf{- 4} \mathbf{x}=\mathbf{0}$, por un factor que contiene la variable.

\section{Grado de una ecuación:}

El grado de una ecuación con una sola incógnita, es el mayor exponente al que está elevado la incógnita en esa ecuación.

Así,

$\mathbf{x}+\mathbf{7}=\mathbf{3} \quad$ es una ecuación de primer grado.

$\mathbf{x}^{\mathbf{2}}+\mathbf{3 x}-\mathbf{2}=\mathbf{0} \quad$ es una ecuación de segundo grado.

$\mathbf{x}^{\mathbf{3}}+\mathbf{5} \mathbf{x}^{\mathbf{2}} \mathbf{- 4} \mathbf{x}=\mathbf{2}$ es una ecuación de tercer grado.

\section{Recursos}

Diapositivas Interactivas

\section{Actividades \\ Ejercicios Varios:}

1.- De las siguientes igualdades, determinar cuáles son identidades y cuales son ecuaciones.
1. $2(x+4)=2 x+8$
2. $2 x-4-2 / 3 x=4 / 3 x-4$
3. $3(x-2)=2(x-3)+x$
4. $x(x+2)=x^{2}+2 x$
5. $x^{2}-5 x+6=(x-1)(x+6)$

2.- En la siguiente lista de ecuaciones reconocer cuales de ellas son equivalentes, defectuosas o redundantes a la ecuación: $\mathbf{3} \mathbf{x}^{\mathbf{2}}+\mathbf{6} \mathbf{x}=\mathbf{0}$.
1. $3 x^{2}+6 x$
2. $x=-2$
3. $x^{2}=-2 x$
4. $x^{2}(x+1)=-2 x(x+1)$ 
3.- Resuelve las siguientes ecuaciones:

1. $7 x-4 x=5-3$

2. $2 x-5 x=2+4$

3. $5 x-2 x=3-11$

4. $4 x-5 x=-2+3$

4.- Resuelve las siguientes ecuaciones:

\section{1. $3 x-2=3-4 x$}

2. $2 x-5=2 x+4$

\section{3. $7 x-5=3-11 x$}

\section{Autoevaluación}

Determinar si las siguientes proposiciones son verdaderas (v) o falsas (f).

a) La expresión: $\mathbf{2 x - 5}=\mathbf{3 x}-\mathbf{2}$ es una igualdad

b) La ecuación: $\mathbf{3 ( x - 2 )}=\mathbf{5}(\mathbf{x}-\mathbf{3})$ es de grado 1 .

c) Las ecuaciones: $\mathbf{x}(\mathbf{x}-\mathbf{2})=\mathbf{0} \mathbf{y} \mathbf{x}^{\mathbf{2}}-\mathbf{2} \mathbf{x}=\mathbf{0}$ son equivalentes.

d) La expresión: $\mathbf{x}+\mathbf{y}=\mathbf{0}$ es una ecuación de grado 2 .

e) La ecuación: $\mathbf{x}^{\mathbf{2}}(\mathbf{x}+\mathbf{5})=(\mathbf{x}+\mathbf{5})(\mathbf{x}-\mathbf{3})$ : es redundante de la ecuación: $\mathbf{x}^{\mathbf{2}}=\mathbf{x}-\mathbf{3}$

f) La ecuación $x^{2}=x-3$ es defectuosa de la ecuación $\left.x^{2}(x+5)=\right)=(x+5)(x-3)$.

g) Una raíz de la ecuación: $2 x+5=x^{3}+1$ es $x=0$.

h) En la ecuación: $\mathbf{3} \mathbf{x}+\mathbf{5}=\mathbf{x}^{\mathbf{2}}-\mathbf{2}$, al pasar el término $\mathbf{x}^{\mathbf{2}}$ del segundo al primer miembro, se obtiene, $x^{2}=\mathbf{4} / 3 x-1$

\section{Reflexión sobre lo aprendido:}

¿Cuándo se hace referencia al concepto ecuación, puedes notar que están implícitos otros conceptos? ¿Puedes recordar cuales y el significado de cada uno?

¿Equidad e igualdad están relacionadas?

Para encontrar las raíces o soluciones de una ecuación, se debe tener en cuenta un par de procedimientos. ¿A qué procedimientos nos referimos?

¿La solución de toda ecuación admite que las mismas sean equivalentes? ¿Si las ecuaciones tomadas no son equivalentes, que nombre reciben?

\section{Conclusión}


El objeto de aprendizaje presentado, está asentado en un repositorio de materiales con el nombre de laboratorio de materiales educativos. Bajo los Modelos: a) Solicitud de servicios, b) Acta de inicio del proyecto de producción, c) Estructura didáctica del objeto de aprendizaje y, d) Planilla para la catalogación de un objeto de aprendizaje.

Con este material dispuesto en la plataforma con el nombre de objeto de aprendizaje (OA), estimula al estudiante a desempeñar la actividad con mayor autonomía, razón por la cual el docente se ve en la obligación de realizar la selección previa del contenido dada una acertada selección de la bibliografía a utilizar.

\section{Referencias Bibliográficas}

Ausubel, D. (1961a): Psicología educativa. Un punto de vista cognoscitivo. Editorial Trillas. México

Ausubel, D. (1960). The use of advance organizers in the learning and retention of meaningful verbal material. Journal of Educational Psychology, 51, 267-272. Disponible: http://es.wikipedia.org/wiki/David_Ausubel [Consulta, 2012, septiembre 2,]

Documento: políticas y estrategias para el desarrollo de la Educación Superior en Venezuela. 20002006. Ministerio de educación cultura y deportes. Diciembre 2001

Documento: Programa Nacional de Formación en Informática (CTPNFI). Caracas, Noviembre 2008. MPPEU/DVDA/DGC PNFI/10/36-178

Garduño, R. (2011). Objetos de aprendizaje en la educación virtual: una aproximación en bibliotecología. Revista scielo, investig. Bibl Vol. 20, No. 41. México. Disponible en: http://www.scielo.org.mx/scielo.php?script=sci_arttext\&pid=S0187-358X2006000200008

López, C. (2011) Los repositorios de objeto de aprendizaje como soporte para los entornos e-learning. Universidad de Salamanca. España. Disponible http://www.biblioweb.tic.unam.mx/libros/repositorios/index.htm [Consulta, Enero 08, 2018]

López, F. A. (2013). Hacer visible nuestro repositorio digital. En: infotecarios. Disponible en: http://www.infotecarios.com/hacer-visible-nuestro-repositorio-digital/ [Consulta. Enero, 09, 2018] 
Miguez, A. (2012). El aula, los alumnos y el profesor de matemáticas. Revista: Enseñanza de la Matemática, Vol. 11, No. 1, 2012, pp. 3-9

Rivera, C. (2014). Características y ventajas de los OA. Disponible en: http://www.infotecarios.com/objetos-de-aprendizaje-una-primera-mirada/ [Consulta, Enero, 08, 2018] 